\title{
Recurrent peripheral facial nerve palsy
}

\section{Parálisis facial periférica recurrente}

\author{
Belén Gómez-González, ${ }^{1}$ Javier González-García, ${ }^{1}$ Ángela Gómez-Farpón, ${ }^{2}$ Rafael Pardo-De la Vega, ${ }^{1}$ \\ Julio Félix-Muñiz, ${ }^{3}$ Begoña Fernández-Martínez, ${ }^{1}$ Ana González-Acero, ${ }^{1}$ Francisco Álvarez-Caro ${ }^{1}$
}

\begin{abstract}
Background: Melkersson-Rosenthal syndrome is a rare disorder that is characterized, in its full form, by recurrent facial nerve palsy, fissured tongue, and oro-facial edema. Most cases present as oligosymptomatic or monosymptomatic forms. Its etiology is still unknown and its course is chronic and it may be progressive.

Case report: We present the case of a nine-year-old girl with recurrent episodes of peripheral facial nerve palsy. During the study, lip edema, benign migratory glossitis, and angular cheilitis were observed, which is why a clinical diagnosis of Melkersson-Rosenthal syndrome was made.

Conclusions: This syndrome must be considered in the differential diagnosis with the presence of acute peripheral facial nerve palsy and/or facial edema due to its behavior and progressive evolution.
\end{abstract}

Key words: Facial palsy; Fissured tongue; Angular cheilitis

Este artículo debe citarse como: Gómez-González B, González-García J, Gómez-Farpón Á, Pardo-De la Vega R, Félix-Muñiz J, Fernández-Martínez B, et al. Parálisis facial periférica recurrente. Rev Alerg Mex. 2020;67(4):421-424

ORCID

Belén Gómez-González, 0000-0003-1372-3809; Javier González-García, 0000-0002-3747-9727;

Ángela Gómez-Farpón, 0000-0001-6819-6837; Rafael Pardo-De la Vega, 0000-0002-6855-6158;

Julio Félix-Muñiz, 0000-0002-1440-7237; Begoña Fernández-Martínez, 0000-0001-8055-1395;

Ana González-Acero, 0000-0002-0207-8384; Francisco Álvarez-Caro, 0000-0003-3049-960X

\begin{abstract}
${ }^{1}$ Hospital Universitario de Cabueñes, Servicio de Pediatría, Gijón, España ${ }^{2}$ Hospital Universitario Central de Asturias, Servicio de Cirugía Pediátrica, Oviedo, España

${ }^{3}$ Hospital Universitario de Cabueñes, Servicio de Otorrinolaringología, Gijón,

España
\end{abstract}

Correspondencia: Belén Gómez González.

belengomezglez@gmail.com

Recibido: 2020-07-17

Aceptado: 2020-09-01

DOI: 10.29262/ram.v67i4.791 


\title{
Resumen
}

Antecedentes: El síndrome de Melkersson-Rosenthal es una entidad poco frecuente caracterizada, en su forma completa, por parálisis facial recurrente, lengua fisurada y edema orofacial. La mayoría de los casos se presentan como formas oligosintomáticas y monosintomáticas. Su etiología es aún desconocida y tiene un curso crónico que puede ser progresivo.

Caso clínico: Presentamos el caso de una niña de nueve años de edad con episodios recurrentes de parálisis facial periférica. Durante su estudio se observó edema labial, glositis migratoria benigna y queilitis angular, por lo que se formuló el diagnóstico clínico de síndrome de MelkerssonRosenthal.

Conclusión: Debemos considerar este síndrome dentro del diagnóstico diferencial ante la presencia de parálisis facial periférica recurrente o edema facial, debido a su comportamiento y evolución progresiva.

Palabras clave: Parálisis facial; Lengua fisurada; Queilitis angular

\author{
Abreviaturas y siglas \\ SMR, síndrome de Melkersson-Rosenthal
}

\section{Antecedentes}

El síndrome de Melkersson-Rosenthal (SMR) es una entidad clínica poco frecuente de etiología desconocida, caracterizada por tres signos clínicos: parálisis facial idiopática recurrente, lengua fisurada y edema orofacial.

La aparición de la tríada clásica es infrecuente (aproximadamente en un tercio de los casos); por lo general se diagnostican formas oligosintomáticas o monosintomáticas de la enfermedad. ${ }^{1}$

La forma monosintomática más común se conoce como queilitis granulomatosa de Meischer y se presenta con edema labial recurrente, de uno o ambos labios, que puede llegar a ser persistente.

El SMR fue descrito por primera vez en 1928 por Melkersson, quien describió episodios recurrentes de parálisis facial acompañados de edema facial. En 1931, Rosenthal añadió la presencia de lengua fisurada entre las características más importantes de este trastorno. ${ }^{2}$

Se han propuesto diferentes mecanismos patogénicos, tales como hipersensibilidad a alimentos, interacción con agentes infecciosos que la activan o atopia; sin embargo, su etiología es aún incierta. ${ }^{3}$

A continuación se describe el caso de una niña afectada por este síndrome.

\section{Caso clínico}

Se presenta el caso de una paciente de nueve años que fue ingresada a urgencias pediátricas por un episodio de parálisis facial aguda izquierda periférica. Entre sus antecedentes médicos destacó la presencia de alergias (a ácaros, epitelio de gato, gramíneas y kiwi) y asma bien controlada, sin tratamiento preventivo. Se administró tratamiento con corticoides orales, tras los cuales hubo mejoría. Seis meses después fue valorada nuevamente por un episodio de características similares que le imposibilitaba cerrar el ojo izquierdo y reducía la movilidad de la hemicara izquierda (grado IV según la escala House-Brackmann, figura 1). No se informaron antecedentes infecciosos, traumáticos o de familiares que hubieran padecido episodios similares.

En la exploración física detallada se observó lengua fisurada con marcados surcos tranversales, macroglosia y edema labial discreto; asimismo, se comprobó queilitis angular (figura 2). La paciente no presentó aftas ni otras lesiones en la mucosa oral. El resto de la exploración física, incluyendo la exploración neurológica, fue normal.

Se solicitaron análisis complementarios, entre los que se incluyeron hemograma, bioquímica y estudio de $\mathrm{C} 1$ inhibidor; los resultados no mostraron 


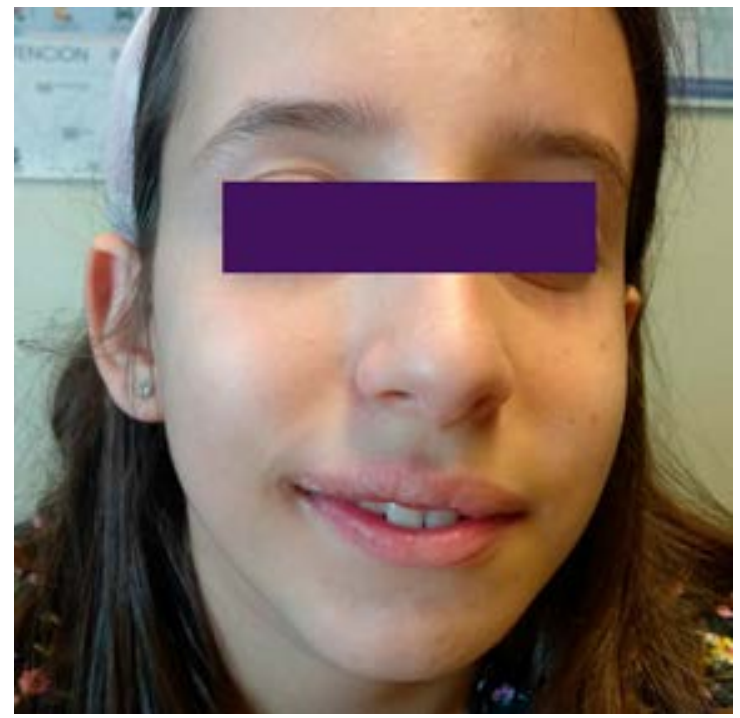

Figura 1. Parálisis facial izquierda con desviación de la comisura labial.

alteraciones ni resultados serológicos de Borrelia burgdorferi; además, se descartó infección por virus de la inmunodeficiencia humana, Mumps ortho rubulavirus (parotiditis), citomegalovirus, virus de Epstein-Barr y herpesvirus tipos 1 y 2. Debido al cuadro clínico se diagnosticó SMR.

Ante nuevo episodio se inició un ciclo de corticoides orales en esquema descendente durante tres semanas, con el cual hubo mejoría progresiva. Se solicitó estudio neurofisiológico en el que, pasados cinco meses, se apreció el comienzo de la reinervación. De igual forma se inició rehabilitación coadyuvante.

En cuanto al seguimiento posterior, la paciente no presentó nuevos episodios y la recuperación era completa al momento de este informe.

\section{Conclusiones}

El SMR no es frecuente en los niños. Se estima que su prevalencia es de aproximadamente $0.08 \%$ en la población general, aunque probablemente esté infradiagnosticado. ${ }^{4}$ Afecta de forma similar a uno y otro sexo, aunque con ligero predominio en las mujeres; generalmente se presenta en la segunda década de la vida.

$\mathrm{Su}$ etiología continúa siendo desconocida. Se han postulado teorías en relación con la autoinmunidad, agentes infecciosos (tales como Borrelia burgdorferi, Mycobacterium tuberculosis, herpesvirus y

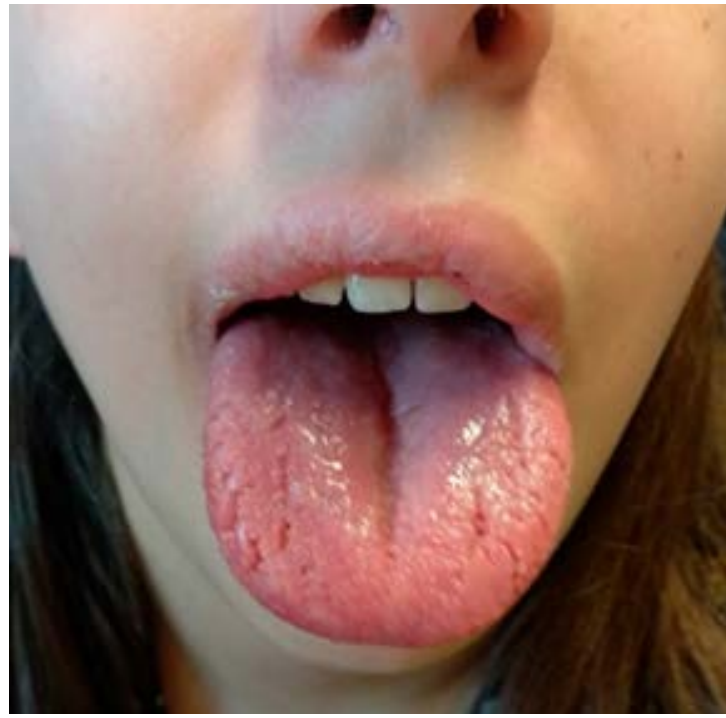

Figura 2. Lengua depapilada, con presencia de surcos tranversales en dorso, compatibles con lengua fisurada. Discreta macroglosia y leve edema de hemilabio superior izquierdo.

espiroquetas), antecedentes personales de alergias, así como causas genéticas. En este sentido, existe un solapamiento entre el SMR, la enfermedad de Crohn y la sarcoidosis. En algunos casos por estudios histológicos se ha observado la misma reacción granulomatosa en las tres entidades. ${ }^{5}$

El cuadro clínico completo se manifiesta en aproximadamente un tercio de los casos y las formas oligosintomáticas y monosintomáticas son las más frecuentes. Debe tenerse en cuenta la posibilidad de la aparición secuencial de los signos y síntomas, por lo que el seguimiento resulta imprescindible. En las formas oligosintomáticas, la manifestación más común es la queilitis granulomatosa de Meischer, que consiste en la tumefacción labial que afecta de forma característica al labio superior. ${ }^{6}$ Habitualmente la duración es de horas a días, con resolución completa de manera inicial, aunque puede hacerse persistente y dolorosa. También puede presentarse descamación, fisuración en comisuras, grietas o quemazón.

La parálisis facial es indistinguible de la parálisis de Bell o parálisis idiopática. ${ }^{7}$ Puede ser unilateral o bilateral, parcial o completa. En la literatura están descritas parálisis alternas y homolaterales en cada episodio. La parálisis de Bell recurre en menos 
de $10 \%$ de los casos, mientras que en el SMR recurre en la mayoría de los sujetos.

En los pacientes pediátricos con SMR se han descrito síntomas como acúfenos, migraña o disgeusia, con afectación ocasional de otros nervios craneales. El diagnóstico es eminentemente clínico; ante duda para establecerlo de esta forma se deberá realizar biopsia de la región afectada para la confirmación anatomopatológica.

Si bien no se dispone de guías clínicas específicas respecto al tratamiento, se han realizado distintas aproximaciones terapéuticas. En ocasiones hay recuperación espontánea del episodio, pero se recomienda optar por iniciar el tratamiento con corticoides, ya sea aplicados directamente a las lesiones o administrados de forma sistémica. Se han propuesto otras opciones terapéuticas en las que se adiciona minociclina, agentes antivirales o gammaglobulinas intravenosas, aunque son necesarios nuevos estudios para valorar la eficacia de estos. En los casos resistentes a tratamiento médico puede ser necesario plantear la descompresión quirúrgica del nervio facial.

\section{Referencias}

1. Wehl G, Rauchenzauner M. A systematic review of the literature of the three related disease entities cheilitis granulomatosa, orofacial granulomatosis and Melkersson-Rosenthal syndrome. Curr Pediatr Rev. 2018;14(3):196-203. DOI: 10.2174/1573396314666180515113941.

2. Kakimoto C, Sparks C, White AA. Melkersson-Rosenthal syndrome: a form of pseudoangioedema. Ann Allergy Asthma Immunol. 2007;99(2):185-189. DOI: 10.1016/S1081-1206(10)60643-6.

3. Jamil RT, Agrawal M, Gharbi A, Sonthalia S. Cheilitis granulomatosa [Internet]. EE. UU.: StatPearls Publishing; 2021. Statpearls. Disponible en: https://www.ncbi.nlm.nih.gov/books/NBK470396/

4. Ziem PE, Pfrommer C, Goerdt S, Orfanos CE, Blume-Peytavi U. Melkersson-Rosenthal syndrome in childhood: a challenge in differential diagnosis and treatment. Br J Dermatol. 2000;143(4):860-863. DOI: 10.1046/j.1365-2133.2000.03791.x

5. Bordino L, Juchli ML, Fernández M, Fitz-Maurice MA, Martins A, Ramírez ZE, et al. Melkersson Rosenthal syndrome. Report of two pediatric cases. Arch Argent Pediatr. 2016;114(4):e224-227. DOI: 10.5546/ aap.2016.e224.

6. Martínez-Menchón T, Mahiques L, Pérez-Ferriols A, Febrer I, Vilata JJ, Fortea JM, et al. Síndrome de Melkersson-Rosenthal. Actas Dermosifiliogr. 2003;94(3):180-183. Disponible en: https://www. actasdermo.org/index.php?p=revista\&tipo=pdf-simple\&pii=13045979

7. Savasta S, Rossi A, Foiadelli T et al. Melkersson-Rosenthal syndrome in childhood: report of three paediatric cases and a review of the literature. Int J Environ Res Public Health. 2019;16(7):1289. DOI: 10.3390/ijerph16071289 